\title{
Evaluación de la competitividad internacional en el contexto global actual: un análisis input-output para el sector industrial
}

\author{
Raúl Vázquez López*
}

Universidad Nacional Autónoma de México, Instituto de Investigaciones Económicas, Circuito Mario de la Cueva s/n, Ciudad Universitaria, 04510 Ciudad de México, Coyoacán, México

Recibido: 24 de enero de 2021 / Aceptado: 21 de abril de 2021

\begin{abstract}
Resumen
Este artículo estudia el desempeño comercial de los 12 principales países exportadores de bienes industriales en el período 2004-2013, analizando tanto la pretendida asociación existente entre la productividad laboral y la competitividad externa como el rol de las industrias altamente tecnológicas en el éxito exportador. La aplicación de la metodología de Wang, Wei y Zhu (2018), a partir del uso de matrices input-output globales para el caso del comercio de Estados Unidos (EE.UU.) y de China, muestra el mejor posicionamiento de EE.UU. en la Cadena Gobal de Valor (CGV), y ejemplifica la relevancia de las nuevas dinámicas productivas globales para la evaluación del comercio internacional a nivel sectorial.
\end{abstract}

\section{Palabras clave}

Industria; comercio internacional; productividad; cadena global de valor; input-output.

\section{Evaluation of international competitiveness and insertion in global value chains: An analysis input-output for the industrial sector}

\begin{abstract}
This article examines the commercial performance of the top 12 industrial goods exporting countries in 2004-2013, analyzing both the purported partnership between labour productivity and external competitiveness, and the role of highly technological industries in export success. The application of Wang, Wei, and Zhu's methodology (2018), based on the use of global inputproduct matrices, for U.S. and Chinese trade, shows the best U.S. positioning in Global Value Chain (GVC) and exemplifies the relevance of new global productive dynamics for the evaluation of international trade at the sectoral level.
\end{abstract}

\section{Keywords}

Industry; international trade; productivity; global value chain; input-output.

JEL Codes: C67, F10, L60, 010.

\footnotetext{
* Correspondencia autor: rvazquez@unam.mx
} 


\section{Introducción}

En las últimas décadas, el fenómeno de la globalización económica, apuntalado por los avances en materia tecnológica, se ha traducido en una nueva división internacional del trabajo (NDIT) articulada alrededor de complejas redes globales de producción que involucran a industrias de un gran número de países. La segmentación del proceso de fabricación en tareas que implican diferentes contenidos factoriales y el empleo de trabajadores de diversos grados de cualificación, así como la posterior deslocalización geográfica de estas actividades, han configurado un funcionamiento jerárquico en el que las industrias nacionales se insertan de forma diferenciada. En esta NDIT, los principales actores son los entes coordinadores de cadenas globales de valor, generalmente casas matrices de grandes empresas transnacionales, responsables de maximizar la rentabilidad del conjunto de la cadena y, en consecuencia, de la dirección y coordinación de una amplia variedad de actividades productivas realizadas en distintas localizaciones.

Como resultado, y debido, entre otros aspectos, a las brechas existentes en términos de costes salariales y niveles de productividad laboral entre los países, las tareas intensivas en trabajo de escasa cualificación, ligadas a los procesos de fabricación, se han desplazado paulatinamente desde las naciones industriales tradicionales hacia países emergentes en Asia y América Latina, con China asumiendo el papel de centro manufacturero mundial y Estados Unidos como principal mercado de consumo. A pesar de esta profunda transformación, la mayor inserción global y la creciente competitividad externa de las industrias en estas nuevas localizaciones no se ha traducido en una captura proporcional de los beneficios de la actividad, en razón de las diferencias existentes en materia de tareas efectuadas, de políticas gubernamentales relacionadas con la repatriación de los beneficios, de nacionalidad de la propiedad de las empresas y de la naturaleza del gobierno corporativo en contextos nacionales específicos (Henderson, Dicken, Hess, Coen y Yeung, 2002).

Mientras ciertos países emergentes se han internado en procesos de especialización productiva, a partir de la realización de tareas de ensamblaje para la exportación y con reducidos efectos de arrastre sobre el resto del aparato fabril, otros países han aumentado la dependencia de sus ciclos económicos en relación con la producción y la exportación de bienes intensivos en recursos naturales y/o de commodities industriales, cuyos precios internacionales son altamente volátiles. A este respecto, existen diversas propuestas para incrementar tanto los beneficios obtenidos de la realización de tareas productivas como los niveles de competitividad externa de las industrias en estas nuevas localizaciones. Algunos autores, dando continuidad a enfoques clásicos, argumentan sobre la necesidad de incrementar los grados de eficiencia productiva a nivel de planta; por su parte, otras reflexiones, de corte principalmente heterodoxo, sostienen la pertinencia de una escalada productiva en los perfiles nacionales de exportación en la dirección de bienes e industrias de mayor intensidad tecnológica.

En este contexto, el presente artículo, en calidad de primer trabajo exploratorio de la problemática expuesta, tiene como principal objetivo analizar la correlación existente entre el éxito exportador y distintos factores teóricos explicativos de la competitividad externa, tales como la productividad laboral, la participación de industrias de alto contenido tecnológico en las exportaciones y los grados de especialización productiva, para el caso de los 12 principales países exportadores de bienes industriales del mundo. Los hallazgos muestran que, de forma agregada, no parece existir relación entre los grados de productividad laboral y los saldos comerciales registrados, como tampoco parece verificarse el rol de las industrias tecnológicamente avanzadas como motor de la competitividad externa. Estos resultados se explican en gran medida por el comercio de bienes, teóricamente paradójico, entre las dos principales potencias económicas, Estados Unidos y China, cuyo saldo comercial encuentra su origen en las dinámicas particulares de la NDIT. A la luz de estos resultados, un segundo objetivo de este artículo es destacar la pertinencia teórico-metodológica de evaluar la competitividad externa a nivel sectorial a partir del contenido en conocimiento de las tareas efectivamente realizadas en las distintas localizaciones, y no en función del tipo y de la cantidad de bienes exportados, como se ha hecho tradicionalmente.

La estructura de este artículo es la siguiente. En la sección 2 se sintetizan las distintas reflexiones teóricas que se van a evaluar, destacando los indicadores utilizados en cada caso para estudiar la 
competitividad externa a nivel sectorial: saldo en la balanza comercial, productividad laboral, diversificación de las exportaciones y peso de las industrias tecnológicamente intensivas. En la sección 3 se detalla la construcción de las bases de información, las fuentes de datos, y se resumen los aspectos metodológicos de los distintos ejercicios realizados. A continuación, en la sección 4 se presentan, de forma descriptiva y en calidad de desafío a las reflexiones teóricas antes mencionadas, los resultados de los ejercicios en términos tanto de la pretendida asociación existente entre la productividad laboral y la competitividad externa, como del rol de las industrias altamente tecnológicas en el éxito exportador. El caso paradigmático del comercio de Estados Unidos y China se aborda en la sección 5, introduciendo una evaluación de este a partir del valor agregado doméstico contenido en los intercambios efectuados. Por último, se concluye con la necesidad de contar con una visión de la competitividad externa, cuya conceptualización e instrumentos de análisis partan de la comprensión de las formas jerárquicas de organización y funcionamiento del sistema productivo global actual, de forma que se establezcan alineamientos alternativos de inserción internacional para los países que buscan desarrollar sus economías a través del comercio exterior.

\section{Referencias teóricas y estudios sobre la relación productividad-competitividad}

En los enfoques tradicionales de la competitividad externa, el concepto vincula la capacidad de las empresas de un país para vender productos o servicios en el extranjero con causas macroeconómicas, tales como los precios relativos, los costes laborales y los niveles de los tipos de cambio. No obstante, como señalan Karadeloglou y Benkovskis (2015), existe evidencia robusta en lo relativo a la contribución de otros factores en la determinación de los grados de competitividad externa, entre los que destacan, en particular, los niveles de productividad y la integración en cadenas globales de valor, así como factores estructurales específicos de cada caso. Entre los trabajos que explican la competitividad nacional, utilizando indicadores económicos agregados y esencialmente a partir de la evaluación del desempeño de un país en el contexto de la competencia internacional, las variables de análisis utilizadas suelen ser el saldo en la balanza comercial, las participaciones en el comercio mundial y el grado de penetración de las importaciones en los mercados nacionales.

Chudnovsky y Porta (1990) argumentan que el aumento en la productividad, en particular en la laboral, es la condición necesaria, pero no suficiente, para incrementar la competitividad. A este respecto, los análisis suelen diferenciar, por una parte, una competitividad "auténtica", basada en ventajas dinámicas y relacionada con mejoras en la productividad sustentadas en el cambio tecnológico, así como en una transformación estructural progresiva de los patrones de industrialización, de su forma "espuria", asociada con ventajas de tipo estático, como el coste de la mano de obra, las variaciones del tipo de cambio y una diversa gama de cuestiones, como la localización geográfica, que pueden influir en el tipo y en la intensidad de la demanda externa. De hecho, Padilla (2006) define la competitividad efímera, artificial o espuria como la asociada con bajos salarios, explotación no sustentable de recursos naturales, condiciones laborales inadecuadas y, fundamentalmente, basada en ventajas comparativas estáticas. Por su parte, la competitividad real o auténtica es la asociada en su presentación con el incremento de la capacidad productiva de las nuevas formas de organización empresarial, y está basada en ventajas comparativas dinámicas que permiten aumentar los salarios y el nivel de vida de la población.

Para los autores que estudian la competitividad de un país a partir de variables pertenecientes al ámbito sectorial, la definición implícita está asociada con una ventaja absoluta o relativa en términos de productividad, al fabricar los bienes comerciados (Gächter, Lorenz, Ramskogler y Silgoner, 2013; Máñez, Rochina-Barrachina y Sanchís-Llopis, 2015). Mientras que para algunos trabajos las ventas al extranjero aceleran los procesos de industrialización e incrementan el tamaño del mercado disponible para las empresas, permitiéndoles una mayor explotación de las economías de escala y, en consecuencia, grados crecientes de eficiencia (Atkin, Khandelwal y Osman, 2017; Máñez et al., 2015), otros estudios empíricos para diferentes casos sostienen que son las empresas más productivas las que logran introducirse en 
los mercados foráneos, siendo ese el sentido de la relación de causalidad del aumento de la productividad en relación con los avances en competitividad (Altuzarra, Bustillo y Rodríguez, 2016; Tadesse, White y Shukralla, 2015).

A modo de ejemplo, la mayoría de los estudios sobre la relación productividad-competitividad externa en los países relevantes para este artículo se centran tanto en la posible existencia de dicho vínculo como en el sentido de la causalidad entre los dos conceptos, utilizando datos provenientes de encuestas a nivel de planta o de industria. En su artículo, considerado decisivo para el tema, Bernard y Jensen (1999) encuentran, en el caso del auge exportador de Estados Unidos entre 1987 y 1994, un impacto reducido del comercio exterior sobre la productividad, a partir de regresiones de los cambios de las ventas al exterior, y de las importaciones sobre el tipo de cambio, la demanda externa y las medidas de productividad. En el trabajo complementario de Bernard y Jensen (2004) sobre el caso, con información de los censos de manufacturas del país, las variaciones en el tipo de cambio y el incremento en los ingresos externos son responsables de la mayor parte del aumento de las ventas al exterior, mientras el crecimiento de la eficiencia de las plantas desempeña un rol menor.

En esta línea de ideas, Bao y Blanco (2014) señalan que en los mercados internacionales no solo se enfrentan entre sí empresas sino redes de vinculaciones entre centros educativos, infraestructuras tecnológicas, relaciones gerenciales-laborales, aparatos institucionales y sistemas financieros, entre otros. Desde esta perspectiva, algunos estudios de la competitividad externa de los países han subrayado la importancia de la diversificación productiva y de los procesos de innovación en la determinación de una competitividad externa de tipo estructural (Agosín, 2009; Hausmann y Klinger, 2006; Klinger y Lederman, 2006). En el enfoque del espacio-producto elaborado por Hausmann y Klinger (2006), la competitividad internacional y el progreso económico de una nación se producen como resultado de la escalada en la producción y exportación de bienes dirigidos a productos relacionados con un mayor grado de sofisticación. La mayor flexibilidad con que las capacidades y habilidades acumuladas pueden ser redireccionadas de un sector a otro, como consecuencia de la diversificación, se constituyen en externalidades interindustriales que hacen más densa la estructura productiva en su conjunto.

Con independencia del sentido de la relación entre los niveles de eficiencia y la competitividad externa, así como de otros determinantes de esta, en el contexto de la NDIT actual diversos estudios han subrayado que la evolución competitiva de un país o de una región está condicionada por su tipo de inserción en CGV y, en definitiva, por su trayectoria anterior, en un contexto internacional desigual tanto en términos de desarrollo económico como de regulación institucional (Banga, 2013; Sheppard, 2017). Como señala Banga (2013), una mayor participación en CGV puede estar asociada a ganancias en términos de acceso a mercados globales, expansión de la producción, mejoras en la eficiencia productiva como resultado de la difusión de tecnología de punta, y creación de empleos. Sin embargo, a día de hoy, las herramientas para medir el grado de participación de un país en CGV, así como la distribución de los ingresos generados por esa participación, son limitadas. En definitiva, lo que los países exportan en términos de bienes puede ser muy diferente de lo que realmente aportan al proceso de producción de sus exportaciones en términos de valor agregado. De este modo, países grandes exportadores de bienes industriales pueden contribuir con muy poco valor a las exportaciones realizadas, lo que afecta, en particular, a la correcta evaluación del grado de sofisticación de estas (Johnson, 2014).

En el marco analítico de las CGV, el concepto de gobernanza ilustra la centralización del poder de decisión en un ente coordinador que determina cómo se distribuyen en el interior de estos ordenamientos las tareas, las responsabilidades y los beneficios, así como los recursos financieros, materiales y humanos que se van a invertir en cada fase (Kaplinsky, 2000) ${ }^{1}$. Las barreras a la entrada de los segmentos estratégicos de mayor rentabilidad de la cadena permiten a las empresas líderes que los ocupan, generalmente empresas matrices de grupos transnacionales, el control de los encadenamientos hacia atrás y hacia delante del proceso productivo. Los elementos de control del conjunto de la red, en manos del

${ }^{1}$ Kaplinsky (2000, p. 124) define el concepto de gobernanza como el "papel de coordinación y de identificación de oportunidades dinámicas de realización de rentas, así como la asignación de actividades a los diferentes participantes en el proceso de producción". 
ente coordinador, cambian en función de la naturaleza de los escalones, y varían desde la posesión de determinados activos tecnológicos, la especificación del producto, la disponibilidad de canales de distribución, la fijación de los precios de venta de los bienes e insumos, hasta aspectos tales como la financiación y las diferentes capacidades de gestión (Comisión Económica para América Latina y el Caribe [CEPAL], 2008).

Esta planificación empresarial transnacional, cuyo criterio es la maximización del beneficio privado, determina colateralmente estructuras productivas, niveles salariales $y$, en definitiva, grados de bienestar y desarrollo locales, regionales y nacionales (CEPAL, 2004). Como muestra la teoría de la "curva de la sonrisa" (Baldwin, Ito y Sato, 2014; Mudambi, 2008; Ye, Meng y Wei, 2015), los distintos países, dependiendo de su posición y grado de participación en CGV, es decir, de su tipo de inserción en estos ordenamientos internacionales, exhiben niveles muy diferentes de ganancias en términos de ingresos, de valor agregado y de empleo. Debido a las ventajas comparativas presentes en las distintas localizaciones, los países avanzados participan en la cadena realizando actividades intangibles de producción tales como la investigación y el desarrollo, el diseño y la elaboración de la marca en las etapas de prefabricación, y el comercio y los servicios de posventa en las etapas posteriores a la fabricación. Por el contrario, los países menos avanzados se insertan en estas cadenas de forma subordinada por la vía de la ejecución de tareas de manufactura y ensamblaje, que implican bajos niveles de contenido en valor agregado y en salarios.

En relación con ello, diversos autores (Ahmad y Primi, 2017; Taglioni y Winkler, 2016) han adoptado recientemente el crecimiento del valor añadido doméstico en las exportaciones brutas como principal indicador de una inserción más favorable en las CGV. Una mayor participación del valor agregado doméstico en las exportaciones industriales resulta de la creación y del fortalecimiento de las relaciones entre empresas nacionales y, en general, de los grados de articulación entre sectores productivos locales (Taglioni y Winkler, 2016). Además, dado que los trabajadores altamente cualificados suelen especializarse en actividades de mayor valor añadido, tales como las tareas de investigación y desarrollo, diseño y comercialización y, últimamente, en servicios posventa, de elevados niveles de ingresos (Low y Pasadilla, 2016), una proporción creciente de valor doméstico suele indicar tanto un mejor posicionamiento de una empresa/industria/país determinado en estas CGV, como la generación de empleos mejor remunerados (Tian, Dietzenbacher y Jong-A-Pin, 2019). En síntesis, como señala Bair (2005), se concluye la necesidad de incluir en el análisis de la competitividad externa los mecanismos regulatorios y las propiedades estructurales del capitalismo contemporáneo que determinan la configuración y la operación de estas cadenas.

\section{Metodología y datos}

Desde una perspectiva crítica, este trabajo busca destacar, en el caso de los 12 principales países exportadores de bienes industriales del mundo en el año 2013, la ausencia de correlación aparente entre el éxito exportador y distintos factores explicativos teóricos de la competitividad externa, tales como la productividad laboral, la participación de industrias de alto contenido tecnológico en las exportaciones y los grados de especialización productiva, profundizando en el comercio de bienes, teóricamente paradójico, entre Estados Unidos y China. Con este objetivo, se utilizan y consolidan múltiples fuentes de información. La productividad laboral se calcula como el cociente del valor agregado entre el número de empleados para las 119 ramas de la industria a nivel de 4 dígitos, bajo la CIIU Rev. 3, a partir de los datos obtenidos de la INDSTAT4 2012 elaborada por la Organización de las Naciones Unidas para el Desarrollo Industrial (UNIDO). Con el fin de subrayar los datos relevantes en materia de una posible asociación productividad-competitividad, se separan los 12 países en dos grupos: uno de alta productividad, formado por los seis países con los niveles más altos en el indicador en 2004, y otro de baja productividad, en el que se encuentran las seis naciones con los niveles de eficiencia comparativamente más bajos en dicho año. 
Para hacer compatible esta información, proveniente de las encuestas industriales de los distintos países y con los datos de comercio exterior, obtenidos de la base COMTRADE elaborada por la Organización de las Naciones Unidas (ONU) y descargada utilizando el programa WITS del World Bank bajo la clasificación SA88/92, se procede a reclasificar los 4.511 productos identificados en función de las 119 ramas a 4 dígitos de la clasificación CIIU Rev. 3. Se calculan, entonces, las importaciones, las exportaciones, la balanza comercial y los coeficientes de exportaciones sobre importaciones para las 119 ramas, para el total del sector, para los grupos de productividad antes delineados y para cuatro tipos de industrias (grupos de ramas) en función de su contenido tecnológico, empleando la clasificación de industrias por conocimiento incorporado elaborada por la Organisation for Economic Co-operation and Development (OECD) (tecnología alta, tecnología medio-alta, tecnología medio-baja y tecnología baja) (OECD, 2005) (ver Tabla 1).

Tabla 1. Clasificación de industrias de la OCDE por I+D incorporado

\begin{tabular}{|c|c|c|c|}
\hline \multirow[t]{2}{*}{ ISIC Rev. 3} & \multicolumn{2}{|r|}{ WIOD (ISIC Rev. 4) } & \multirow[b]{2}{*}{ Código } \\
\hline & Código & & \\
\hline \multicolumn{4}{|l|}{ Industrias de alta tecnología } \\
\hline Aeroespacial. & 353 & $\begin{array}{l}\text { Manufactura de productos farmacéuticos } \\
\text { básicos y preparaciones farmacéuticas. }\end{array}$ & $\mathrm{C} 21$ \\
\hline Productos farmacéuticos. & 2423 & Equipos de cómputo, electrónico y de óptica. & $\mathrm{C} 26$ \\
\hline Computadoras y máquinas de oficinas. & 30 & & \\
\hline Equipos de telecomunicación. & 32 & & \\
\hline Instrumental médico e instrumental óptico. & 33 & & \\
\hline \multicolumn{4}{|l|}{ Industrias de tecnología medio-alta } \\
\hline Máquinas y aparatos eléctricos. & 31 & Química y productos químicos. & $\mathrm{C} 20$ \\
\hline Vehículos a motor, trailers y semitrailers. & 34 & Equipo eléctrico. & $\mathrm{C} 27$ \\
\hline Productos químicos, excluidos los farmacéuticos. & $\begin{array}{l}24 \text { excl. } \\
2423\end{array}$ & Maquinaria y equipo n.e.c. & $\mathrm{C} 28$ \\
\hline Equipo de transporte n.e.c. & $352+359$ & Vehículos de motor, trailers y semitrailers. & $\mathrm{C} 29$ \\
\hline Máquinas y equipos. & 29 & Otro equipo de transporte. & C30 \\
\hline \multicolumn{4}{|l|}{ Industrias de tecnología medio-baja } \\
\hline Construcción y reparamiento de barcos y botes. & 351 & $\begin{array}{l}\text { Coque, productos de refinamiento de } \\
\text { petróleo. }\end{array}$ & C19 \\
\hline Productos plásticos. & 25 & Plástico y productos de plástico. & $\mathrm{C} 22$ \\
\hline Coque, productos del refinamiento de petróleo. & 23 & Otros productos de minerales no metálicos. & $\mathrm{C} 23$ \\
\hline Productos de minerales no metálicos. & 26 & Metales básicos. & $\mathrm{C} 24$ \\
\hline Metales básicos y productos metálicos elaborados. & $27-28$ & $\begin{array}{l}\text { Productos fabricados de metal excluyendo } \\
\text { maquinaria y equipo. }\end{array}$ & $\mathrm{C} 25$ \\
\hline \multicolumn{4}{|l|}{ Industrias de baja tecnología } \\
\hline Manufacturas n.e.c. reciclados. & $36-37$ & Alimentos, bebidas y tabaco. & C10-C12 \\
\hline $\begin{array}{l}\text { Madera, productos de papel, impresos y } \\
\text { publicaciones. }\end{array}$ & $20-22$ & Textiles, vestido y productos de cuero. & C13-C15 \\
\hline Productos alimenticios, bebidas y tabaco. & $15-16$ & $\begin{array}{l}\text { Madera y productos de madera y corcho, } \\
\text { excluyendo muebles. }\end{array}$ & $\mathrm{C} 16$ \\
\hline \multirow[t]{3}{*}{ Productos textiles, cuero y calzado. } & $17-19$ & Papel y productos de papel. & $\mathrm{C} 17$ \\
\hline & & $\begin{array}{l}\text { Impresiones y reproducción de medios } \\
\text { grabados. }\end{array}$ & $\mathrm{C} 18$ \\
\hline & & Muebles y otras manufacturas. & C31-C32 \\
\hline
\end{tabular}

Fuente: elaboración propia a partir de OECD (2005) y World Input-Output Database (WIOD, 2020). 
La cobertura temporal y por ramas de la información es muy dispar en los diferentes casos, por lo que es necesario limitarse al estudio de un período y centrar las observaciones en la comparación de sus años iniciales y finales. Con el fin de contar con un lapso de tiempo lo suficientemente largo para registrar la evolución reciente de los procesos de interés, y evitar años afectados por eventos económicos internacionales relevantes que pudieran sesgar el análisis tales como crisis o recesiones globales, se opta por calcular los distintos indicadores para el período 2004-2013.

$E^{S r}=\underbrace{\left(V^{S} B^{S S}\right)^{T} \# Y^{S r}}_{(1)}+\underbrace{\left(V^{S} B^{S S}\right)^{T} \#\left(A^{S r} B^{r r} Y^{r r}\right)}_{(2)}+$

$+\underbrace{\left(V^{S} L^{S S}\right)^{T} \#\left[A^{s r} \sum_{t \neq s, r}^{G} B^{r t} Y^{t t}+A^{s r} B^{r r} \sum_{t \neq s, r}^{G} Y^{r t}+A^{s r} \sum_{t \neq s, r}^{G} B^{r t} \sum_{u \neq s, t}^{G} Y^{t u}\right]}+$

(3)

$+\underbrace{\left(V^{S} L^{S S}\right)^{T} \#\left[A^{s r} B^{r r} Y^{r s}+A^{s r} \sum_{t \neq s, r}^{G} B^{r t} Y^{t s}+A^{s r} B^{r s} Y^{s s}\right]}+$

(4)

$+\underbrace{\left[\left(V^{S} L^{s S}\right)^{T} \#\left(A^{s r} B^{r s} \sum_{t \neq s}^{G} Y^{s t}\right)+\left(V^{s} L^{s s} \sum_{t \neq s}^{G} A^{s t} B^{t s}\right)^{\mathrm{T}} \#\left(A^{s t} X^{r}\right)\right]}+\underbrace{\left[\left(V^{r} B^{r s}\right)^{\mathrm{T}} \# Y^{s r}+\left(\sum_{t \neq s, \mathrm{r}}^{G} V^{t} B^{t s}\right)^{T} \# Y^{s r}\right]}+$ (5)

(6)

$\underbrace{+\left[\left(V^{r} B^{r s}\right)^{T} \#\left(A^{s r} L^{r r} Y^{r r}\right)+\left(\sum_{t \neq s, r}^{G} V^{t} B^{t s}\right)^{T} \#\left(A^{s r} L^{r r} Y^{r r}\right)\right]}+\underbrace{\left[\left(V^{r} B^{r s}\right)^{T} \#\left(A^{s r} L^{r r} E^{r^{*}}\right)+\left(\sum_{t \neq s, r}^{G} V^{t} B^{t s}\right)^{T} \#\left(A^{s r} L^{r r} E^{r^{*}}\right)\right]}$ (7)

El componente (1) calcula el valor agregado de origen doméstico contenido en las exportaciones de bienes finales del país $s$ consumidas por el importador directo (país $r$ ); el componente (2) corresponde al valor agregado doméstico contenido en las exportaciones de bienes intermedios del país $s$, usadas por el importador directo $r$ para la producción de bienes finales; el componente (3) calcula el valor agregado doméstico utilizado por el importador directo (país $r$ ) para producir bienes exportados que serán consumidos por terceros países; y el componente (4) se corresponde con el valor agregado doméstico contenido en las exportaciones de bienes intermedios que regresan y son consumidas en el país $s$.

La suma de estos cuatro primeros componentes proporciona el valor agregado doméstico contenido en las exportaciones brutas del país $r$ al país o grupo de países $s$ (VAX). Por su parte, los componentes (6) y (7) representan el valor agregado extranjero contenido en las exportaciones realizadas (VAE). De igual forma, mientras el sumatorio de los términos (3) y (4) proporciona el valor agregado doméstico incluido en la exportación de bienes intermedios para su reexportación por parte de terceros países (IV), los componentes (5) y (8) registran la doble contabilidad en el ejercicio.

Una vez calculados los VAX, VAE y el IV para el caso del comercio de bienes industriales entre Estados Unidos y China para los años 2004 y 2013 a nivel de los 22 sectores industriales de la clasificación CIIU Rev. 4 presentes en la base de información, se suman los datos obtenidos en grupos de industrias y se calculan las participaciones de estos conceptos en el valor total de las exportaciones brutas tanto en el total de industrias como para las industrias de tecnología alta y media alta $(A+M A)$. La Tabla 1 muestra 
la reagrupación de industrias realizada, en función del contenido tecnológico incorporado, siguiendo de nuevo la clasificación de la OECD.

\section{Resultados: productividad laboral y competitividad externa}

En la Tabla 2 se presentan los niveles de productividad laboral para el total de la industria y para el conjunto de industrias de A+MA en el año inicial de nuestro período de estudio, en el caso de las naciones seleccionadas, y separándolas en dos grupos en función de sus grados de eficiencia. Se calcula, asimismo, para el indicador un índice base 100 referente a Estados Unidos en 2004. Por su parte, en la Tabla 3, se recogen los valores de las exportaciones, de las importaciones y de la balanza comercial en 2004 y en 2013 para los 12 países y los grupos conformados tanto para el total de la industria como para el conjunto de industrias de tecnología alta y media-alta.

Tabla 2. Productividad laboral en los países seleccionados, 2004 (en dólares corrientes)

\begin{tabular}{lccccc}
\hline & \multicolumn{2}{c}{ Total industria } & & \multicolumn{2}{c}{ Industrias A+MA } \\
\cline { 2 - 3 } \cline { 5 - 6 } País & Productividad & Índice & & Productividad & Índice \\
\cline { 2 - 3 } Estados Unidos & 154.544 & 100 & & 193.871 & 100 \\
Japón & 125.209 & 81 & & 153.107 & 79 \\
Corea & 102.277 & 66 & & 116.702 & 60 \\
Bélgica & 99.315 & 64 & & 119.513 & 62 \\
Holanda & 79.951 & 52 & & 87.621 & 45 \\
Reino Unido & 78.968 & 51 & & 88.965 & 46 \\
Media alta productividad & 106.711 & 69 & & 126.630 & 65 \\
Alemania & 73.129 & 47 & & 82.210 & 42 \\
Francia & 69.398 & 45 & & 78.722 & 41 \\
Italia & 66.290 & 43 & & 71.430 & 37 \\
Singapur & 64.761 & 42 & & 75.922 & 39 \\
México & 57.504 & 37 & & 69.241 & 36 \\
China & 9.775 & 6 & & 11.665 & 6 \\
Media baja productividad & 56.810 & 37 & & 64.865 & 33 \\
Promedio del total & 81.760 & 53 & & 95747 & 49 \\
\hline
\end{tabular}

Fuente: elaboración propia con datos de UNIDO (2019), INDSTAT4 Rev. 3.

A nivel de países, Estados Unidos muestra una productividad laboral más alta tanto en la industria en su conjunto como al considerar solamente las industrias de tecnología alta y media alta. Significativamente, el saldo en la balanza comercial es negativa en ambos casos, y en particular en las actividades tecnológicamente intensivas. Estos resultados reflejan los elevados niveles de consumo y, en consecuencia, de las importaciones en la economía estadounidense. A grandes rasgos, lo anterior se explica, en línea con lo señalado por Bernard y Jensen (2004), por un comercio exterior determinado más por el manejo del tipo de cambio, y otras variables macroeconómicas, que por los grados de eficiencia a nivel de planta. Las estimaciones realizadas confirman también lo señalado por Inklaar, O'Mahony y Timmer (2005) en cuanto a los niveles de productividad laboral superiores en la industria de Estados Unidos con respecto a sus competidores europeos, pero con brechas mayores en nuestros resultados, en particular en las industrias A+MA.

Los antecedentes empíricos muestran que, efectivamente, la ventaja comparativa de los Estados Unidos está basada en un patrón de especialización intensivo en conocimiento con mayores grados de intensidad del capital y con una dotación superior de recursos humanos cualificados. No obstante, en el contexto de la fragmentación y de la deslocalización geográfica de segmentos productivos, bajo la 
gobernanza de las grandes empresas transnacionales norteamericanas, el país registra saldos negativos en su balanza comercial asociados tanto a la manipulación del tipo de cambio del dólar como al auge de las importaciones intraindustriales. La ausecia de correlación entre los niveles de productividad laboral y la competitividad externa se expresa de forma clara tanto a nivel de algunos países, cuyas dinámicas son de sobra significativas, como en el ámbito de los grupos delineados. Mientras la productividad en el grupo de países más eficientes es superior a la del segundo grupo en un $80 \%$ para el total de la industria, y en un 95\% para las industrias A+MA, su saldo comercial es inferior a la de la agrupación de países con menor productividad relativa en ambos casos (ver Tabla 3).

Tabla 3. Exportaciones, importaciones y balanza comercial de países seleccionados, 2004 y 2013 (en millones de dólares corrientes)

\begin{tabular}{|c|c|c|c|c|c|c|}
\hline \multirow{3}{*}{ País } & \multicolumn{6}{|c|}{ Total industria } \\
\hline & \multicolumn{2}{|c|}{ Exportaciones } & \multicolumn{2}{|c|}{ Importaciones } & \multicolumn{2}{|c|}{ Balanza comercial } \\
\hline & 2004 & 2013 & 2004 & 2013 & 2004 & 2013 \\
\hline EE.UU. & 645,937 & $1,094,589$ & $1,268,483$ & $1,892,078$ & $-622,546$ & $-797,489$ \\
\hline Japón & 537,643 & 669,083 & 332,080 & 515,513 & 205,562 & 153,570 \\
\hline Corea & 252,258 & 556,266 & 169,221 & 333,766 & 83,037 & 222,500 \\
\hline Bélgica & 275,172 & 454,462 & 243,250 & 392,056 & 31,923 & 62,406 \\
\hline Holanda & 252,669 & 493,044 & 217,101 & 406,221 & 35,568 & 86,822 \\
\hline Reino Unido & 295,208 & 451,850 & 428,081 & 529,502 & $-132,872$ & $-77,652$ \\
\hline Sumatorio alta productividad & $2,258,887$ & $3,719,294$ & $2,658,216$ & $4,069,137$ & $-399,329$ & $-349,843$ \\
\hline Alemania & 829,026 & $1,335,861$ & 565,631 & 934,640 & 263,395 & 401,221 \\
\hline Francia & 382,942 & 515,474 & 378,505 & 567,341 & 4,436 & $-51,867$ \\
\hline Italia & 336,753 & 478,004 & 290,397 & 361,847 & 46,356 & 116,157 \\
\hline Singapur & 190,695 & 373,643 & 156,976 & 320,092 & 33,719 & 53,551 \\
\hline México & 159,330 & 316,073 & 184,267 & 353,758 & $-24,938$ & $-37,685$ \\
\hline China & 576,665 & $2,177,477$ & 436,089 & $1,140,743$ & 140,577 & $1,036,734$ \\
\hline Sumatorio baja productividad & $2,475,411$ & $5,196,532$ & $2,011,866$ & $3,678,421$ & 463,545 & $1,518,111$ \\
\hline \multirow[t]{2}{*}{ Total } & $4,734,297$ & $8,915,827$ & $4,670,081$ & $7,747,559$ & 64,216 & $1,168,268$ \\
\hline & \multicolumn{6}{|c|}{ Industrias $\mathrm{A}+\mathrm{MA}$} \\
\hline \multirow[t]{2}{*}{ País } & \multicolumn{2}{|c|}{ Exportaciones } & \multicolumn{2}{|c|}{ Importaciones } & \multicolumn{2}{|c|}{ Balanza comercial } \\
\hline & 2004 & 2013 & 2004 & 2013 & 2004 & 2013 \\
\hline EE.UU. & 484,068 & 679,208 & 775,186 & $1,180,604$ & $-291,119$ & $-501,396$ \\
\hline Japón & 444,065 & 511,166 & 176,775 & 278,447 & 267,290 & 232,719 \\
\hline Corea & 175,003 & 373,109 & 106,688 & 196,756 & 68,315 & 176,353 \\
\hline Bélgica & 168,723 & 264,031 & 153,319 & 234,174 & 15,404 & 29,857 \\
\hline Holanda & 155,790 & 264,377 & 142,190 & 227,941 & 13,600 & 36,436 \\
\hline Reino Unido & 200,172 & 240,310 & 259,208 & 290,691 & $-59,036$ & $-50,381$ \\
\hline Sumatorio alta productividad & $1,627,821$ & $2,332,201$ & $1,613,367$ & $2,408,613$ & 14,454 & $-76,412$ \\
\hline Alemania & 594,133 & 948,427 & 359,371 & 566,577 & 234,762 & 381,849 \\
\hline Francia & 250,352 & 330,994 & 224,395 & 324,785 & 25,957 & 6,209 \\
\hline Italia & 172,080 & 242,682 & 168,822 & 193,176 & 3,258 & 49,506 \\
\hline Singapur & 151,467 & 256,685 & 119,569 & 192,271 & 31,899 & 64,413 \\
\hline México & 119,334 & 241,538 & 122,257 & 232,863 & $-2,923$ & 8,675 \\
\hline China & 314,994 & $1,232,120$ & 325,020 & 853,980 & $-10,026$ & 378,140 \\
\hline Sumatorio baja productividad & $1,602,359$ & $3,252,445$ & $1,319,433$ & $2,363,653$ & 282,926 & 888,792 \\
\hline Total & $3,230,180$ & $5,584,646$ & $2,932,800$ & $4,772,265$ & 297,380 & 812,381 \\
\hline
\end{tabular}

Fuente: elaboración propia con datos de ONU (2019) y de COMTRADE, descargado del programa WITS del World Bank (2019). 
Es de señalar que sintomáticamente en Estados Unidos y en Reino Unido los elevados niveles de productividad del trabajo se dan a la par de altos niveles de importaciones, particularmente en las industrias A+MA (62\% del total de las importaciones en Estados Unidos en 2103). Con las excepciones de Japón, Corea y Alemania, el conjunto de naciones consideradas en los ejercicios no registra una balanza comercial favorable en las industrias A+MA, por lo que los resultados indican una paradójica correlación negativa entre éxito exportador y peso de las industrias A+MA en las ventas al exterior.

Un caso interesante de estudio es el de México, país que registra en el período una mejora en su saldo comercial externo en las ramas A+MA y un importante deterioro de este mismo saldo a nivel del total de la industria. La ausencia de efectos de arrastre de las industrias tecnológicamente intensivas hacia el resto de la estructura de fabricación, fruto de un esquema de especialización productiva basado en el ensamblaje de insumos y componentes importados, pone en duda el papel, dependiente del tipo de inserción en CGV, de estas industrias en un proceso sustantivo de competitividad auténtica (Vázquez, 2013).

\section{Valor agregado incorporado en las exportaciones de China y Estados Unidos}

En este orden de ideas, aunque una explicación genérica del fenómeno está relacionada con las diferencias en la intensidad en capital presentes en las distintas actividades y, en consecuencia, con los grados de diversificación y con la composición de las ventas al exterior de los casos de estudio, lo cierto es que las particularidades de las dinámicas comerciales de Estados Unidos y China son de especial interés. En términos de productividad laboral, la relación entre EE.UU. y China fue de 12 a 1 en favor de la economía norteamericana (ver Tabla 2). No obstante, tras un fuerte crecimiento de las exportaciones en los diferentes títulos, prueba de un desempeño competitivo apuntalado por un elevado grado de diversificación y a la vez de sofisticación, en 2013 las exportaciones industriales de China duplicaron el valor de las del país norteamericano, alcanzando los 2.177 mil millones de dólares (de este importe, el 16,9\% fueron ventas dirigidos a Estados Unidos).

La Figura 1 compara la estructura de las exportaciones, en términos de su contenido tecnológico en los años 2004 y 2013, en China y en EE.UU. Los cálculos agregados muestran un cambio regresivo en la composición de las ventas al extranjero de EE.UU., de tal modo que las exportaciones de las industrias de alta tecnología cayeron desde representar el 33\% al 19\% del total. En el caso del país asiático, se destaca, en cambio, la persistencia de un modelo de crecimiento exportador diversificado, con una importante presencia de las industrias de alta tecnología en las ventas al exterior (33\% del total en 2013). Se puede afirmar, entonces, que el exitoso desempeño comercial de China, en parte apuntalado por las industrias $\mathrm{A}+\mathrm{MA}$, se produce en un marco de baja productividad del trabajo y de menores costes laborales relativos, es decir, de ventajas competitivas estáticas. Por su parte, la escasa competitividad externa de EE.UU. está relacionada, paradójicamente, con un patrón de especialización industrial basado en elevados niveles de eficiencia y, en suma, en ventajas competitivas dinámicas, lo que refuta la esencia del conjunto de reflexiones teóricas tradicionales analizadas.

Un elemento explicativo infravalorado por la literatura económica en lo referente a la aparente ausencia de relación existente entre la competitividad externa y sus principales determinantes teóricos reside en las formas jerárquicas de organización y de funcionamiento de una NDIT capitaneada por entes coordinadores de CGV que asignan a las industrias de los distintos países tareas diferenciadas en términos de sus contenidos factoriales. En este nuevo contexto, las tareas intensivas en conocimiento generadoras de altos niveles de beneficio, anteriormente en su mayoría constitutivas del proceso de fabricación, son realizadas en calidad de servicios (diseño, mercadotecnia, gestión o distribución, entre otras) en los centros industriales tradicionales localizados en países desarrollados. Lo anterior constituye un proceso de dos caras: una desindustrialización en favor del desarrollo de actividades de servicios en los países avanzados y, paralelamente, como resultado de la deslocalización geográfica de 
segmentos productivos, una industrialización generalmente asociada a la especialización en tareas de bajo contenido en valor agregado en naciones emergentes ${ }^{2}$.

\section{Estados Unidos 2004}

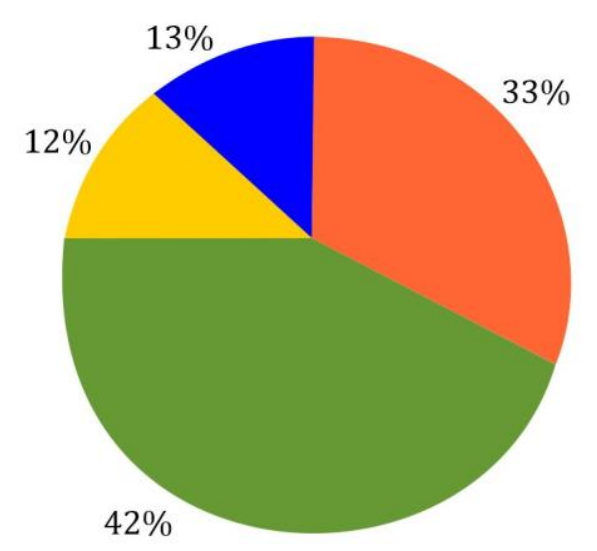

$\because 1 \quad 2 \quad \square \quad \square 4$

Estados Unidos 2013

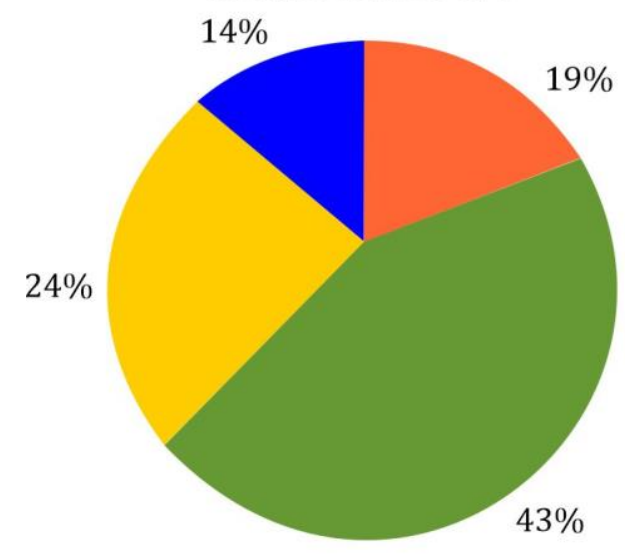

$\because 1 \quad 2 \quad \square \quad \square \quad 4$

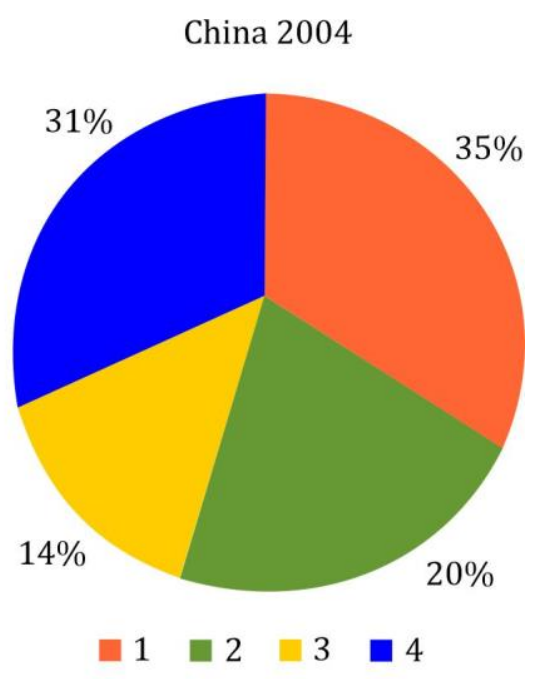

China 2013

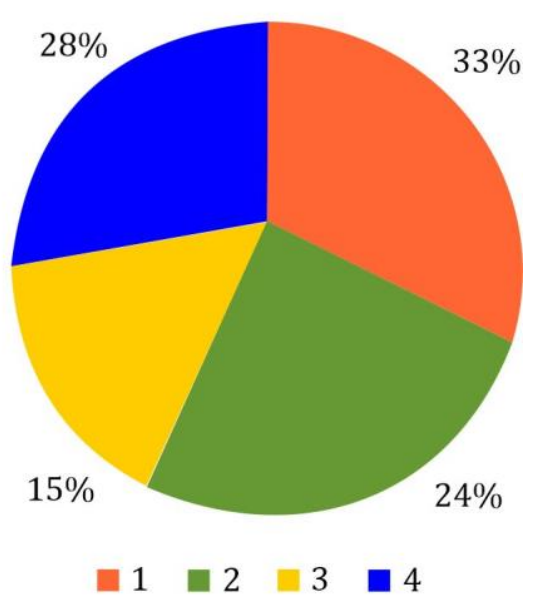

Figura 1. Evolución de la estructura de las exportaciones por contenido tecnológico en Estados Unidos y China, 2004 y 2013 (en porcentajes). Fuente: elaboración propia con datos de la ONU (2019) y de COMTRADE, descargado del programa WITS del World Bank (2019).

Como consecuencia, el precio de los productos intercambiados internacionalmente resulta de la suma de los valores añadidos por las tareas realizadas en diversas localizaciones. En términos de contabilidad, las exportaciones y las importaciones de bienes finales reflejan el coste y los niveles de eficiencia del conjunto de las tareas efectuadas por los distintos participantes de la cadena, y no

2 Es de subrayar que en muchos países emergentes este proceso de especialización productiva, además de reducir los grados de diversificación y la densidad del aparato de fabricación local, es concomitante de una reprimarización de la economía, tras el incremento de los precios de los commodities y la consiguiente apreciación de los tipos de cambio resultantes de la creciente demanda de alimentos y recursos naturales por parte de China. En América Latina, en especial, este fenómeno profundiza la desindustrialización prematura previa (productividad y empleo industriales estancados), asociada a una baja competitividad de los precios de los productos exportados (Bresser-Pereira, 2010; Salama, 2012). 
únicamente los relativos a los segmentos de fabricación realizados en el país exportador (Gereffi y Fernández-Stark, 2016). En definitiva, este contexto global cambiante exige una readecuación teórico-conceptual de los instrumentos y de la información comúnmente utilizada para el análisis del comercio internacional. Un primer paso indispensable en esta dirección son los esfuerzos recientes por contabilizar los intercambios globales en términos del valor añadido en cada etapa del proceso productivo (Borin y Mancini, 2015; Johnson y Noguera, 2012; Wang et al., 2018), en vez de hacerlo en función del valor total acumulado en los bienes comerciados, como se ha hecho hasta ahora.

En este sentido, los resultados de la aplicación de la metodología de Wang et al. (2018) muestran la relevancia del tipo de inserción en CGV para el análisis de la competitividad externa a nivel sectorial. En la Tabla 4 se presentam las participaciones en el valor total de las exportaciones brutas, del valor agregado doméstico (VAX), del valor agregado extranjero (VAE) y del valor agregado doméstico incluido en la exportación de bienes intermedios para su reexportación por parte de terceros países (IV), para el conjunto de la industria y para las industrias A+MA, en el caso del comercio entre China y EE.UU. Estos porcentajes pueden ser interpretados como un primer indicador tanto del grado de sofisticación de las ventas al exterior como, en particular, del posicionamiento de las industrias nacionales en las distintas CGV. Una elevada participación del VAX en las exportaciones brutas resulta de la realización de tareas de mayor contenido tecnológico, con independencia de la industria en cuestión, ya que casi todos los procesos de fabricación pueden ser segmentados en tareas, con grados muy dispares de contenidos factoriales, y en conocimiento.

Tabla 4. Comercio entre Estados Unidos y China en términos de valor agregado (en porcentajes)

\begin{tabular}{|c|c|c|c|c|c|c|}
\hline & \multicolumn{3}{|c|}{$\begin{array}{c}\text { Exportaciones } \\
\text { Estados Unidos - China }\end{array}$} & \multicolumn{3}{|c|}{$\begin{array}{c}\text { Exportaciones } \\
\text { China - Estados Unidos }\end{array}$} \\
\hline & 2004 & 2013 & Media 2004-2013 & 2004 & 2013 & Media 2004-2013 \\
\hline $\begin{array}{l}\text { VAX } \\
\text { Total industria }\end{array}$ & 86,37 & 83,01 & 84,47 & 74,13 & 79,58 & 77,19 \\
\hline $\begin{array}{l}\text { VAX } \\
\text { Industrias A+MA }\end{array}$ & 86,62 & 82,76 & 84,71 & 67,96 & 75,27 & 71,90 \\
\hline $\begin{array}{l}\text { VAE } \\
\text { Total industria }\end{array}$ & 10,27 & 14,61 & 12,43 & 24,74 & 18,67 & 21,49 \\
\hline $\begin{array}{l}\mathrm{VAE} \\
\text { Industrias A+MA }\end{array}$ & 10,17 & 15,14 & 12,40 & 30,64 & 22,41 & 26,46 \\
\hline $\begin{array}{l}\text { IV } \\
\text { Total industria }\end{array}$ & 20,04 & 10,82 & 16,35 & 2,83 & 4,89 & 3,74 \\
\hline $\begin{array}{l}\text { IV } \\
\text { Industrias A+MA }\end{array}$ & 20,26 & 10,17 & 16,71 & 2,98 & 5,91 & 4,20 \\
\hline
\end{tabular}

Nota: VAX, valor agregado doméstico; VAE, valor agregado extranjero; IV, valor agregado doméstico incluido en la exportación de bienes intermedios para su reexportación por parte de terceros países. Fuente: elaboración propia con datos de WIOD (2019).

En suma, elevadas participaciones del VAX reflejan mayores efectos de arrastre de la actividad exportadora y, en definitiva, beneficios más elevados a nivel nacional en términos de producción, de generación de empleo cualificado y de niveles de rentabilidad de la actividad, entre otros. Los hallazgos del ejercicio coinciden con lo señalado a nivel de planta por la mayoría de los estudios empíricos existentes, en cuanto al mayor contenido en conocimiento de las exportaciones de bienes industriales de EE.UU. 
tanto en el conjunto del sector como en las industrias A+MA. Hay que subrayar, sin embargo, el rápido incremento reciente de las participaciones del valor agregado doméstico contenido en las exportaciones chinas, y la tendencia a la baja de estos mismos porcentajes en el caso de EE.UU. La consiguiente reducción de la brecha existente en estas participaciones entre estos dos países confirma el paulatino deterioro de la competitividad externa de Estados Unidos y el documentado ascenso de la economía china.

Koopman, Powers, Wang y Wei (2010) apuntan, a su vez, la relevancia de comparar el peso del valor agregado doméstico incluido en las exportaciones sectoriales de bienes intermedios, realizadas por un país y utilizadas por terceros países de cara a sus propias ventas al extranjero (IV), con el uso de insumos intermedios importados para la exportación de bienes (VAE). De tal forma que si un país se inserta en los segmentos iniciales de la cadena (upstream), usualmente de mayores niveles tecnológicos, a partir de la venta de insumos para la reexportación de bienes por parte de terceros países, el peso de su IV tiende a ser más alto, mientras que el de su VAE tiende a ser más bajo. Por el contrario, si el país se introduce en CGV de manera menos favorable, a través de tareas de ensamblaje de productos finales, intensivas en empleo de baja cualificación, y a partir de la utilización de insumos importados intermedios (downstream), el peso de su VAE suele ser mayor y el de su IV, más reducido.

A partir de este razonamiento, los resultados obtenidos para el comercio entre Estados Unidos y China confirman claramente un posicionamiento más favorable de las industrias de Estados Unidos en los segmentos iniciales de las cadenas (upstream) con respecto a las industrias chinas, altamente dependientes de la importación de insumos para la exportación y con un contenido marginal de valor agregado doméstico en las exportaciones de bienes intermedios, que son utilizados para la reexportación por terceros países (IV) (4,89\% en 2013). En efecto, en 2004, tanto en las exportaciones totales de bienes industriales como en las relativas a las industrias A+MA, los porcentajes de IV en Estados Unidos eran superiores en aproximadamente siete veces a los registrados por China, mientras que las participaciones del VAE fueron considerablemente mayores en el país asiático $(30,64 \%$ por $10,17 \%$ en el país norteamericano en las industrias A+MA).

A pesar del saldo comercial negativo registrado por Estados Unidos, el mejor posicionamiento cualitativo de sus industrias en CGV, resultante en parte del rol dominante y de la coordinación de las empresas trasnacionales estadounidenses en estas redes jerárquicamente organizadas, permite al país norteamericano obtener beneficios más elevados de su comercio exterior. Sin embargo, se confirma también una rápida reducción de la brecha competitiva entre los dos países. A lo largo del período, el valor agregado extranjero contenido en las exportaciones de Estados Unidos a China registra una marcada tendencia al alza, concomitante con una reducción del valor agregado doméstico reexportado por terceros países (IV). Por el contrario, en las exportaciones de China hacia Estados Unidos se registran tendencias opuestas, con una disminución en la participación del VAE y con un incremento tendencial del valor agregado doméstico reexportado por terceros países (IV). En suma, parece que la hegemonía económica de Estados Unidos ha permitido a sus industrias verse favorecidas con relaciones comerciales asimétricas que, no obstante, han sido cada vez más disputadas por el ascenso de la economía china.

\section{Discusión y conclusiones}

El análisis de la competitividad externa de los 12 principales países exportadores de bienes industriales del mundo proporciona una evidencia contradictoria en lo referente a los determinantes del éxito exportador comúnmente aceptados en la literatura especializada. De forma agregada, a nivel sectorial no parece existir relación entre los grados de productividad laboral y los saldos comerciales registrados, como tampoco parece verificarse el rol de las industrias tecnológicamente avanzadas como motor de la competitividad externa. De hecho, al intentar asociar estadísticamente los índices de productividad laboral calculados para el año 2004 con los coeficientes de exportaciones sobre importaciones de los años 2004 y 2013 para el total de la industria y para las ramas A+MA, los resultados no son significativos y la relación es incluso negativa en tres de los cuatro ejercicios realizados para el conjunto de casos. No 
obstante, estas contradicciones se explican en gran parte por el comercio de las dos principales potencias comerciales, Estados Unidos y China, cuyos saldos comerciales encuentran su origen en las dinámicas particulares de la globalización económica actual.

Por todo ello, si no se consideran los casos atípicos, pero por supuesto trascendentales de Estados Unidos y China, se encuentra una correlación positiva y significativa entre las dos variables, particularmente en las ramas A+MA (ver Figura 2). Otro hallazgo relevante es que el grado de correlación es mayor cuando se asocian los índices de eficiencia de 2004 con los coeficientes del mismo año, y es menor cuando se trata de explicar la competitividad futura (2013). Lo anterior puede indicar que, en el caso de la mayoría de las economías altamente exportadoras de bienes industriales, con las notables excepciones de Estados Unidos y China, de existir una relación entre ambos conceptos esta se produciría en las industrias tecnológicamente intensivas, y probablemente el sentido de esta no sería de la productividad laboral en dirección a la competitividad externa, como sostienen Altuzarra et al. (2016) y Tadesse et al. (2015).
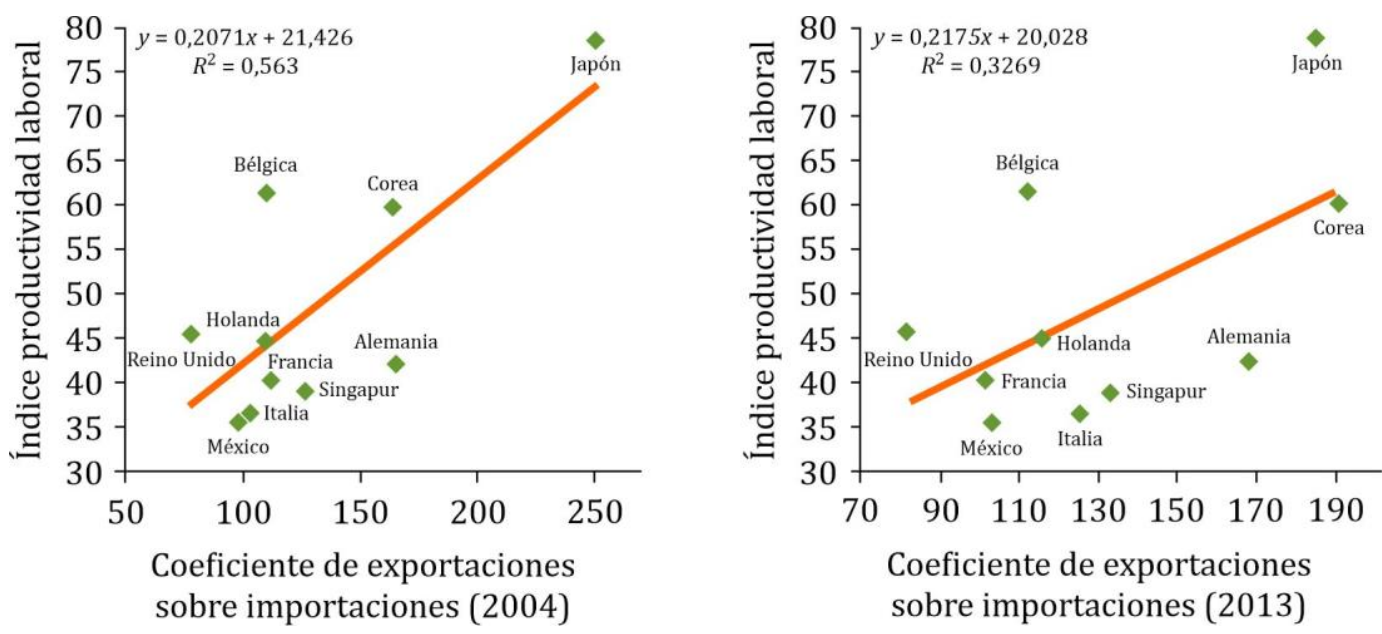

Figura 2. Productividad laboral y coeficiente de exportaciones sobre importaciones en países seleccionados, 2004 y 2013 (índice en dólares corrientes). Fuente: elaboración propia con datos de la ONU (2019), COMTRADE, descargado del programa WITS del World Bank (2019).

En lo referente al comercio de China y Estados Unidos, que en su conjunto (exportaciones e importaciones totales) representa cerca del 50\% del total de la muestra de los 12 países, sus determinantes permiten destacar aspectos recientes de las dinámicas productivas y comerciales globales, cuyo análisis requiere nuevos instrumentos y una profundización de las teorías existentes. Una evaluación de los intercambios comerciales, en términos del valor agregado contenido en las exportaciones, muestra el carácter sistémico y a la vez jerárquico de la competitividad, y pone en evidencia la necesidad de estudiar el comercio y la producción en el ámbito de las tareas desempeñadas y no en el de las industrias a las que pertenecen, lo que apunta a la necesidad de generar estadísticas con un mayor grado de desagregación. En suma, parece que son las tareas de alto contenido en conocimiento, independientemente de la industria en que se realicen, las que pueden ejercer como motor del crecimiento, pero no así cualquier tarea desempeñada en las industrias A+MA.

Los hallazgos de este estudio exploratorio de los determinantes del éxito exportador llevan, entonces, a reformular el conjunto de reflexiones teóricas tradicionales alrededor de la pertinencia de modelos de crecimiento basados en la especialización productiva a partir de la exportación de bienes de alta tecnología. Se hace evidente, en particular, que la evaluación de la competitividad externa, a partir de considerar exclusivamente las cantidades de exportaciones e importaciones, no permite entender aspectos 
fundamentales del comercio actual tales como el tipo de inserción en CGV de cada industria nacional. A este respecto, una perspectiva de interés para los países a la búsqueda de desarrollar sus economías a través del comercio exterior está relacionada con la sustitución de criterios tecnológicos de especialización, descontextualizados y ajenos a las realidades nacionales, en favor de alineamientos de carácter estructural que privilegien la mayor sofisticación de las tareas realizadas, la diversificación del aparato de fabricación y los efectos de arrastre de las tareas exportadoras, con el fin de favorecer la creación de empleos bien remunerados.

\section{Agradecimientos}

Investigación realizada gracias al Programa UNAM-PAPIIT No. IN300120, denominado Inserción global, cambio estructural y escalamiento en Cadenas Globales de Valor: un análisis sectorial para la industria.

\section{Bibliografía}

Agosin, M. R. (2009). Crecimiento y diversificación de exportaciones en economías emergentes. Revista de la CEPAL, 97(1), 117-134. DOI: https://doi.org/10.18356/0e25c429-es

Ahmad, N., y Primi, A. (2017). From domestic to regional to global: Factory Africa and factory Latin America? En World Bank (Ed.), Measuring and analyzing the impact of GVCs on economic development (pp. 69-89). Washington, DC: World Bank. Recuperado de: http://hdl.handle.net/10986/29593

Altuzarra, A., Bustillo, R., y Rodríguez, C. (2016). Understanding export market success: Evidence from Manufacturing firms. Open Economies Review, 27(1), 161-181. DOI: https://doi.org/10.1007/s11079-015-9368-6

Atkin, D., Khandelwal, A. K., y Osman, A. (2017). Exporting and firm performance: Evidence from a randomized experiment. The Quarterly Journal of Economics, 132(2), 551-615. DOI: https://doi.org/10.1093/qje/qix002

Bair, J. (2005). Global capitalism and commodity chains: Looking back, going forward. Competition \& Change, 9(2), 153-180. DOI: https://doi.org/10.1179/102452905X45382

Baldwin, R., Ito, T., y Sato, H. (2014). Portrait of factory Asia: Production network in Asia and its implication for growth -the "smile curve". Joint Research Program Series, No. 159. Tokyo, Japan: Institute of Developing Economies-Japan External Trade Organization (IDE-JETRO). Recuperado de: https://www.ide.go.jp/English/Publish/Reports/Jrp/159.html

Banga, R. (2013). Measuring value in global value chains. Background Paper No. RVC-8. Geneva, Switzerland: UNCTAD. Recuperado de: https://unctad.org/system/files/official-document/ecidc2013misc1 bp8.pdf

Bao, S., y Blanco, F. (2014). Modelos de formación de clústers industriales: revisión de las ideas que los sustentan. Revista Galega de Economía, 23(2), 179-198. DOI: https://doi.org/10.15304/rge.23.2.2488

Bernard, A. B., y Jensen, J. B. (1999). Exceptional exporter performance: Cause, effect, or both? Journal of International Economics, 47(1), 1-25. DOI: https://doi.org/10.1016/S0022-1996(98)00027-0

Bernard, A. B., y Jensen, J. B. (2004). Exporting and productivity in the USA. Oxford Review of Economic Policy, 20(3), 343-357. DOI: https://doi.org/10.1093/oxrep/grh020

Bresser-Pereira, L. C. (2010). Globalization and competition. Cambridge, UK: Cambridge University Press. Recuperado de: http://www.bresserpereira.org.br/documento/3816

Borin, A., y Mancini, M. (2015). Follow the value added: Bilateral gross exports accounting. Temi di Discussione. Working Paper No. 1026. Rome, Italy: Banca d'Italia. Recuperado de: https://www.bancaditalia.it/pubblicazioni/temi-discussione/2015/2015-1026/index.html?com.dotmarketing.htmlpage.language $=1$

CEPAL. (2004). Desarrollo productivo en economías abiertas. Santiago de Chile, Chile: Comisión Económica para América Latina y el Caribe. Recuperado de: https://www.cepal.org/es/publicaciones/13057-desarrollo-productivo-economias-abiertas

CEPAL. (2008). La transformación productiva 20 años después: viejos problemas, nuevas oportunidades. Santiago de Chile, Chile: Comisión Económica para América Latina y el Caribe. Recuperado de: https://www.cepal.org/es/publicaciones/2889-la-transformacion-productiva-20-anos-despues-viejos-problemas-nuevas 
Chudnovsky, D., y Porta, F. (1990). La competitividad internacional: principales cuestiones conceptuales y metodológicas. Documentos de trabajo No. 03/91. Montevideo, Uruguay: Centro de Estudios e Investigación de Postgrado (CEIPOS), Universidad de la República. Recuperado de: https://hdl.handle.net/20.500.12008/2110

Gächter, M., Lorenz, H., Ramskogler, P., y Silgoner, M. (2013). An export-based measure of competitiveness. Monetary Policy \& The Economy, 2, 75-92.

Gereffi, G., y Fernández-Stark, K. (2016). Global value chain analysis: A primer (2nd Edition). Durham, NC: Center on Globalization, Governance \& Competitiveness (CGGC), Duke University.

Hausman, R., y Klinger, B. (2006). Structural transformation and patterns of comparative advantage in the product space. Working Paper No. 128. Cambridge, MA: Center for International Development at Harvard. Recuperado de: http://www.tinyurl.com/y2cs9r9z

Henderson, J. H., Dicken, P., Hess, M., Coe, N., y Yeung, H. W.-C. (2011). Global production networks and the analysis of economic development. Review of International Political Economy, 9(3), 436-464. DOI: https://doi.org/10.1080/09692290210150842

Inklaar, R., O'Mahony, M., y Timmer, M. (2005). Productivity and competitiveness in the EU and the US. En M. O'Mahony y B. van Ark (Eds.), EU productivity and competitiveness: An industry perspective. Can Europe resume the catching-up process? (pp. 73-148). Brussels, Belgium: European Commission. Recuperado de: https://ec.europa.eu/digital-single-market/en/news/eu-productivity-and-competitiveness-industry-perspective-can-europe-resume-catching-process-pdf

Johnson, R. C. (2014). Five facts about value added exports and implications for macroeconomics and trade research. Journal of Economic Perspectives, 28(2), 119-142. DOI: https://doi.org/10.1257/jep.28.2.119

Johnson, R. C., y Noguera, G. (2012). Accounting for intermediates: Production sharing and trade in value added. Journal of International Economics, 86(2), 224-236. DOI: https://doi.org/10.1016/j.jinteco.2011.10.003

Kaplinsky, R. (2000). Globalization and unequalisation: What can be learned from value chain analysis? Journal of Development Studies, 37(2), 117-146. DOI: https://doi.org/10.1080/713600071

Karadeloglou, P., y Benkovskis, K. (Eds.). (2015). Compendium on the diagnostic toolkit for competitiveness. Occasional Paper Series No. 163). Frankfurt, Germany: European Central Bank (ECB). Recuperado de: https://www.ecb.europa.eu/pub/pdf/scpops/ecbop163.en.pdf

Klinger, B., y Lederman, D. (2006). Innovation and export portfolios. Working Paper No. WPS3872. Washington, DC: World Bank. Recuperado de: http://hdl.handle.net/10986/8362

Koopman, R., Powers, W., Wang, Z., y Wei, S.-J. (2010). Give credit where credit is due: Tracing value added in global production chains. Working Paper No. 16426. Cambridge, MA: National Bureau of Economic Research (NBER). DOI: https://doi.org/10.3386/w16426

Low, P. y Pasadilla, G. (2016). Manufacturing-related services - Summary report. En P. Low y G. O. Pasadilla (Eds.), Services in global value chains: Manufacturing-related services (pp. 1-40). Singapore, Singapore: World Scientific Publishing for Asia-Pacific Economic Cooperation Secretariat. DOI: https://doi.org/10.1142/10073

Máñez, J. A., Rochina-Barrachina, M. E., y Sanchís-Llopis, J. A. (2015). The dynamic linkages among exports, $R \& D$ and Productivity. The World Economy, 38(4), 583-612. DOI: https://doi.org/10.1111/twec.12160

Mudambi, R. (2008). Location, control and innovation in knowledge-intensive industries. Journal of Economic Geography, 8, 699-725. DOI: https://doi.org/10.1093/jeg/lbn024

OECD. (2005). Anexo A Science, Technology and Industry Scoreboard 2005. Paris, France: Organisation for Economic Co-operation and Development.

ONU. (2019). COMTRADE. Geneva, Switzerland: United Nations. Recuperado de: http://comtrade.un.org/db/

Padilla, R. (2006). Instrumentos de medición de la competitividad. México, México: CEPAL, Sede Subregional México. Recuperado de: http://www.competitividad.org.do/wp-content/uploads/2009/01/2.1Indicadoresdecompetitividad1.pdf

Salama, P. (2012). Globalización comercial: desindustrialización prematura en América Latina e industrialización en Asia. Comercio Exterior, 62(6), 34-44.

Sheppard, E. (2017). Competition in space and between places. En T. J. Barnes y E. Sheppard (Eds.), A companion to economic geography (pp. 169-186). Hoboken, NJ: Wiley-Blackwell. DOI: https://doi.org/10.1002/9781405166430.ch11

Tadesse, B., White, R., y Shukralla, E. (2015). Production efficiency and the extensive margins of U.S. exporters: An industry-level analysis. Open Economies Review, 26(5), 941-969.

DOI: https://doi.org/10.1007/s11079-015-9346-z

Taglioni, D. y Winkler, D. (2016). Making global value chains work for development. Washington, DC: World Bank. Recuperado de: http://hdl.handle.net/10986/24426 
Tian, K., Dietzenbacher, E., y Jong-A-Pin, R. (2019). Measuring industrial upgrading: Applying factor analysis in a global value chain framework. Economic Systems Research, 31(4), 642-664.

DOI: https://doi.org/10.1080/09535314.2019.1610728

UNIDO. (2019). INDSTAT4 (ISIC Rev. 3) Database. Vienna, Austria: United Nations Industrial Development Organization. Recuperado de: https://stat.unido.org/

Vázquez, R. (2013). Heterogeneidad estructural y sus determinantes en la manufactura mexicana, 1994-2008. Revista de la CEPAL, 109, 125-141. Recuperado de: http://ru.iiec.unam.mx/3692/

Wang, Z., Wei, S.-J., y Zhu, K. (2018). Quantifying international production sharing at the bilateral and sector levels. Working Paper No. 19677. Cambridge, MA: National Bureau of Economic Research (NBER). Recuperado de: https://www.nber.org/papers/w19677

WIOD. (2020). World Input-Output Database 2013 Release. World Input-Output Database. Recuperado de: http://www.wiod.org/release16

World Bank. (2019). World Integrated Trade Solution (WITS). Washington, DC: World Bank. Recuperado de: http://wits.worldbank.org/

Ye, M., Meng, B., y Wei, S. (2015). Measuring smile curves in global value chains. IDE Discussion Paper No. 530. Tokyo, Japan: Institute of Developing Economies-Japan External Trade Organization (IDE-JETRO). Recuperado de: http://hdl.handle.net/2344/1469 